\title{
Overview and detectability of the genetic modifications in ornamental plants
}

\author{
Anne-Laure Boutigny ${ }^{1}$, Nicolas Dohin ${ }^{1}$, David Pornin $^{1}$ and Mathieu Rolland ${ }^{1}$
}

\begin{abstract}
The market of ornamental plants is extremely competitive, and for many species genetic engineering can be used to introduce original traits of high commercial interest. However, very few genetically modified (GM) ornamental varieties have reached the market so far. Indeed, the authorization process required for such plants has a strong impact on the profitability of the development of such products. Considering the numerous scientific studies using genetic modification on ornamental species of interest, a lot of transformed material has been produced, could be of commercial interest and could therefore be unintentionally released on the market. The unintentional use of GM petunia in breeding programs has indeed recently been observed. This review lists scientific publications using GM ornamental plants and tries to identify whether these plants could be detected by molecular biology tools commonly used by control laboratories.
\end{abstract}

\section{Introduction}

Ornamental plants are economically important for the horticultural industry ${ }^{1}$. They are sold all over the world, and used in gardening, landscaping, and floristry as cut flowers ${ }^{2}$. Genetic modification of ornamental plants has been used to introduce original traits of high commercial interest for the producers and/or consumers, such as improved floral anatomy and morphology, new floral color, induced early flowering, enhanced fragrance or longevity, stress tolerance or disease resistance ${ }^{1-6}$. At least 50 ornamental plants have been transformed, the main species including rose (Rosa hybrida), chrysanthemum (Chrysanthemum morifolium), petunia (Petunia hybrida), and carnation (Dianthus caryophyllus) $)^{4}$. However, very few genetically modified (GM) ornamental varieties have obtained the regulatory approval and have reached the market so far. Indeed, the authorization process required for such plants has a strong impact on the profitability of the development of such products. In the database of biotech/GM crops approval of the International Service for the Acquisition of Agri-biotech Applications (ISAAA),

\footnotetext{
Correspondence: Anne-Laure Boutigny (anne-laure.boutigny@anses.fr)

${ }^{1}$ Anses, Plant Health Laboratory, Bacteriology Virology GMO Unit, 7 rue Jean Dixméras, 49044 Angerscedex 01France
}

only three ornamental species are listed: petunia, rose, and carnation. Nineteen GM events have been registered in the ISAAA database for carnation, one GM event for petunia, and two GM events for rose. So far, only flower color-modified varieties of carnation and rose have been released on the market of some countries, depending on their regulation concerning production and/or commercialization of $\mathrm{GMO}^{4,6}$. In Europe, only two GM carnation varieties may currently be marketed as cut flowers. Genetic modification of ornamental plants has also commonly been used by research groups to study the genetics behind metabolic pathways, physiology, development, interactions with pathogens ${ }^{7-11}$. Numerous genetically transformed material of potential commercial interest have been produced. If by any mean, the traceability of such material was lost, it could be considered as non-GM and introduced in breeding programs.

In 2017, transgenic petunia plants were detected on the market in Europe and in the United States ${ }^{6}$. The detected GM events contained the A1 gene from Zea mays L. encoding the enzyme dihydroflavonol reductase, which was first introduced into a mutant petunia defective for this gene ${ }^{12}$. The first transformed plants had been obtained to study the biochemistry and 
genetics of the flavonoid metabolic pathway. These plants also had the particularity of producing flowers of an orange color not previously seen in the genus ${ }^{12}$. Further studies showed that differences in the methylation of the promoters occurring in the progeny of these plants conducted to the attenuation of the orange phenotype $^{13,14}$. Such plants have never been through the authorization process required in the European Union and should not have been commercialized. Petunia plants containing the A1 gene have nevertheless been found on the market. Furthermore, the detected plants showed several phenotypes (unpublished data), suggesting that the transgenic material has been used in breeding programs for several years. In respect to the absence of approval for marketing or cultivation in Europe, numerous GM petunia plants had to be withdrawn from the European market in 2017.

The construct inserted in the genome of the detected GM petunia contained the A1 gene but also a $35 \mathrm{~S}$ promoter (p35S) derived from the Cauliflower mosaic virus $(\mathrm{CaMV})$. This promoter, with the nopaline synthase terminator from Agrobacterium tumefaciens (tNos), and to a lesser extent sequences such as the phosphinothricin acetyltransferase gene from Streptomyces hygroscopicus (bar) or the 5-enolpyruvylshikimate-3-phosphate synthase gene from Agrobacterium tumefaciens sp. strain CP4 (ctp2-cp4epsps), have been recurrently introduced in constructs used for genetic modification of plants. To test whether a sample may or not contain GM plant material, laboratories use these sequences as screening elements ${ }^{15}$. Diagnostic is mostly based on real-time PCR detection targeting the sequences of interest (p35s, tNos... $)^{16}$.

The recent detection of transformed unauthorized petunia developed for research purpose in the 80's demonstrates the risk that represents the potential nonintentional use of research plant material in breeding programs, especially when the transgene provides a trait of commercial interest. The aim of the present review was to assess the kind of GM ornamentals, which have been developed for research purpose and could therefore be found on the market if used involuntarily in breeding programs. Scientific publications related to research programs involving the transformation of ornamental species and for which the introduced trait could confer a commercial advantage compared with varieties on the market were listed. In addition, we investigated if such unauthorized material would be detectable by laboratories testing for the presence of GM plants.

\section{Literature studied}

From previously published reviews and articles in database of peer-reviewed literature, 166 scientific publications related to genetic modifications of ornamental plants have been collected (Table 1). Among these publications, 29 ornamental plants were represented (Table 2); with chrysanthemum (26.7\%), petunia (15.2\%), orchidaceae (6.7\%), rosa (6.7\%), dianthus (5.5\%), and torenia (5.5\%) being the main ones (above 5\%). Among the listed GM events, $88.5 \%$ were transformed via Agrobacterium tumefaciens, 9.1\% via biolistic methods, and 3.0\% via CRISPR/Cas9 (CRISPR: clustered regularly interspaced short palindromic repeats; Cas9: CRISPR-associated protein 9). Among these publications, $15 \mathrm{GM}$ traits of potential commercial interest were identified relating to biotic/abiotic stress resistance and plant attributes (Table 1). Among these traits, the modification of the flower color was the most studied with $29.1 \%$ of the publications (Table 3). Other important GM traits were morphology (12.7\%), longevity (12.1\%), early flowering (8.5\%), fungi and virus resistance $(7.9 \%)$ (Table 3). Modification of ornamental plants with several traits was studied in $10.3 \%$ of the scientific publications.

\section{Overview of GM traits in ornamental plants Biotic stress resistance}

Biotic stress occurs in plants due to damage done by plant pathogens or pests (bacteria, fungi, virus, insects, parasites, nematodes, aphids) during production, storage, distribution, and end-consumer use. Plant growth and yield are strongly influenced by biotic stress. Several ornamental species have been transformed to introduce or enhance resistance to pathogens or pests. Various genes coding for toxins, protease inhibitors, trypsin inhibitors, lectins, etc. have been employed to create pest resistance in plants ${ }^{5}$. As an example, increased resistance to aphids was observed in transgenic chrysanthemum overexpressing transcription factor CmWRKY $48^{17}$ or the protease inhibitor Sea Anemone Equistatin $(\mathrm{SAE})^{18}$. Expression of a cystatin transgene in Lilium longiflorum conferred resistance to the root lesion nematode Pratylenchus penetrans ${ }^{19}$. Resistance against bacterial and fungal diseases has been created by transferring various genes coding for chitinase, glucanase, osmotin, defensin, etc. into various ornamental plants ${ }^{5}$. Transgenic gladiolus expressing a synthetic antimicrobial peptide, D4E1, had an increased resistance to the soilborne fungus Fusarium oxysporum f. sp. gla$\operatorname{dioli}^{20}$. The antimicrobial peptide tachyplesin I (tpnI) expression reduced bacterial proliferation, colonization, and disease symptoms (reduced by $95-100 \%$ ) in transgenic ornithogalum plant tissues ${ }^{21}$. Virus resistance has been generally introduced by transferring coat protein gene of the virus in the plant. As an example, resistance to cucumber mosaic virus (CMV) was enhanced when a defective CMV replicase gene was transferred to the lilium $^{8}$. Disease resistance in ornamental plants is greatly expected as disease symptoms on plants or cut 
Table 1 Overview of GM ornamental plants in scientific publications.

\begin{tabular}{|c|c|c|c|c|c|c|c|c|c|c|c|c|c|c|c|c|c|c|c|c|}
\hline \multirow[b]{3}{*}{ Plant } & \multicolumn{14}{|c|}{ GM traits } & \multirow{2}{*}{\multicolumn{3}{|c|}{ Transformation }} & \multirow{2}{*}{\multicolumn{2}{|c|}{$\begin{array}{l}\text { Transgene } \\
\text { sequences }\end{array}$}} & \multirow[b]{3}{*}{ References } \\
\hline & \multicolumn{8}{|c|}{ Biotic/abiotic stress resistance } & \multicolumn{6}{|c|}{ Plant attributes } & & & & & & \\
\hline & 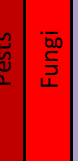 & 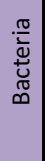 & $\stackrel{n}{\stackrel{n}{5}}$ & 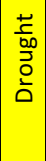 & 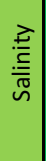 & 흥 & 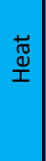 & 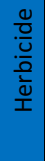 & 흥 & 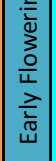 & 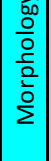 & 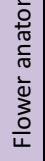 & 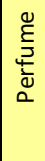 & 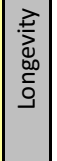 & 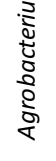 & 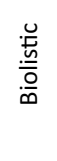 & 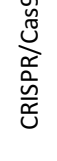 & 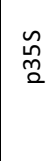 & 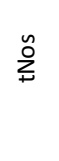 & \\
\hline Anthurium & & & & & & & & & & & & & & & $x$ & & & $\mathrm{x}$ & & 71 \\
\hline \multirow{2}{*}{ Campanula } & & & & & & & & & & & & & & & $x$ & & & & & 56 \\
\hline & & & & & & & & & & & & & & & $x$ & & & & & 57 \\
\hline Celosia & & & & & & & & & & & & & & & $x$ & & & $x$ & & 72 \\
\hline \multirow{44}{*}{ Chrysanthemum } & & & & & & & & & & & & & & & $x$ & & & & $x$ & 73 \\
\hline & & & & & & & & & & & & & & & $x$ & & & $x$ & $x$ & 7 \\
\hline & & & & & & & & & & & & & & & $x$ & & & $x$ & $x$ & 74 \\
\hline & & & & & & & & & & & & & & & $x$ & & & $x$ & $\mathrm{x}$ & 30 \\
\hline & & & & & & & & & & & & & & & $x$ & & & $x$ & $x$ & 75 \\
\hline & & & & & & & & & & & & & & & $x$ & & & $x$ & $x$ & 76 \\
\hline & & & & & & & & & & & & & & & $\mathrm{X}$ & & & $\mathrm{X}$ & & 23 \\
\hline & & & & & & & & & & & & & & & $x$ & & & $x$ & $x$ & 42 \\
\hline & & & & & & & & & & & & & & & $x$ & & & $x$ & & 77 \\
\hline & & & & & & & & & & & & & & & $x$ & & & $x$ & & 47 \\
\hline & & & & & & & & & & & & & & & $\mathrm{x}$ & & & $\mathrm{x}$ & $\mathrm{x}$ & 22 \\
\hline & & & & & & & & & & & & & & & $\mathrm{x}$ & & & $\mathrm{x}$ & & 78 \\
\hline & & & & & & & & & & & & & & & $x$ & & & $x$ & . & 11 \\
\hline & & & & & & & & & & & & & & & $x$ & & & $x$ & $\mathrm{x}$ & 79 \\
\hline & & & & & & & & & & & & & & & $x$ & & & $x$ & $\mathrm{x}$ & 80 \\
\hline & & & & & & & & & & & & & & & $x$ & & & $x$ & $x$ & 45 \\
\hline & & & & & & & & & & & & & & & $x$ & & & $x$ & $x$ & 81 \\
\hline & & & & & & & & & & & & & & & $x$ & & & & & 82 \\
\hline & & & & & & & & & & & & & & & $\mathrm{X}$ & & & $\mathrm{x}$ & $\mathrm{x}$ & 83 \\
\hline & & & & & & & & & & & & & & & $x$ & & & $x$ & $x$ & 17 \\
\hline & & & & & & & & & & & & & & & $x$ & & & $x$ & & 84 \\
\hline & & & & & & & & & & & & & & & $x$ & & & & & 85 \\
\hline & & & & & & & & & & & & & & & $x$ & & & & $x$ & $61 ; 86$ \\
\hline & & & & & & & & & & & & & & & $x$ & & & $x$ & $x$ & 87 \\
\hline & & & & & & & & & & & & & & & $x$ & & & & $x$ & 31 \\
\hline & & & & & & & & & & & & & & & $\mathrm{X}$ & & & $\mathrm{x}$ & & 88 \\
\hline & & & & & & & & & & & & & & & $\mathrm{x}$ & & & & & 89 \\
\hline & & & & & & & & & & & & & & & $x$ & & & & & 90 \\
\hline & & & & & & & & & & & & & & & $\mathrm{x}$ & & & $\mathrm{x}$ & $\mathrm{x}$ & 91 \\
\hline & & & & & & & & & & & & & & & $\mathrm{X}$ & & & $\mathrm{x}$ & & 39 \\
\hline & & & & & & & & & & & & & & & $x$ & & & $x$ & & 92 \\
\hline & & & & & & & & & & & & & & & $x$ & & & $x$ & $x$ & 93 \\
\hline & & & & & & & & & & & & & & & $x$ & & & & $x$ & 94 \\
\hline & & & & & & & & & & & & & & & $\mathrm{x}$ & & & & $\mathrm{x}$ & 95 \\
\hline & & & & & & & & & & & & & & & $x$ & & & & 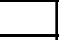 & 96 \\
\hline & & & & & & & & & & & & & & & $x$ & & & $x$ & & 32 \\
\hline & & & & & & & & & & & & & & & $\mathrm{X}$ & & & & $\mathrm{X}$ & 58 \\
\hline & & & & & & & & & & & & & & & $x$ & & & $x$ & $x$ & 24 \\
\hline & & & & & & & & & & & & & & & $x$ & & & $x$ & $x$ & 97 \\
\hline & & & & & & & & & & & & & & & $x$ & & & & & 18 \\
\hline & & & & & & & & & & & & & & & $x$ & & & $x$ & & 46 \\
\hline & & & & & & & & & & & & & & & $x$ & & & & & 40 \\
\hline & & & & & & & & & & & & & & & $x$ & & & $x$ & & 98 \\
\hline & & & & & & & & & & & & & & & $x$ & & & $x$ & & 37 \\
\hline Cyclamen & & & & & & & & & & & & & & & $\mathrm{x}$ & & & $\mathrm{x}$ & & 99 \\
\hline
\end{tabular}




\begin{tabular}{|c|c|c|c|c|c|c|c|c|c|c|c|c|c|c|c|c|c|c|c|c|}
\hline \multirow[b]{3}{*}{ Plant } & \multicolumn{14}{|c|}{ GM traits } & \multirow{2}{*}{\multicolumn{3}{|c|}{ Transformation }} & \multirow{2}{*}{\multicolumn{2}{|c|}{$\begin{array}{l}\text { Transgene } \\
\text { sequences }\end{array}$}} & \multirow[b]{3}{*}{ References } \\
\hline & \multicolumn{8}{|c|}{ Biotic/abiotic stress resistance } & & Plan & at attr & tribu & & & & & & & & \\
\hline & & 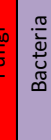 & $\stackrel{n}{5}$ & 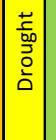 & : & $\frac{0}{0}$ & $\mid \begin{array}{l}\stackrel{+}{\varpi} \\
\mathbb{\Xi}\end{array}$ & $\left|\begin{array}{l}\frac{0}{0} \\
\frac{0}{0} \\
\frac{0}{2} \\
\frac{0}{1} \\
\end{array}\right|$ & 흥 & 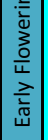 & 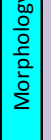 & 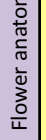 & 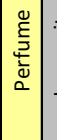 & 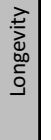 & 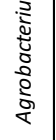 & 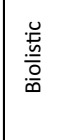 & 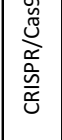 & $\stackrel{\substack{n \\
\alpha}}{a}$ & $\sum_{i}^{n}$ & \\
\hline & & & & & & & & & & & & & & & $\mathrm{x}$ & & & $\mathrm{x}$ & $\mathrm{x}$ & 53 \\
\hline & & & & & & & & & & & & & & & $\mathrm{x}$ & & & $\mathrm{x}$ & & 100 \\
\hline & & & & & & & & & & & & & & & $\mathrm{x}$ & & & $\mathrm{x}$ & & 101 \\
\hline & & & & & & & & & & & & & & & $\mathrm{x}$ & & & $\mathrm{x}$ & $\mathrm{x}$ & 51 \\
\hline Dianthus & & & & & & & & & & & & & & & $\mathrm{x}$ & & & $\mathrm{x}$ & $\mathrm{x}$ & 102 \\
\hline & & & & & & & & & & & & & & & $\mathrm{x}$ & & & & & 62 \\
\hline & & & & & & & & & & & & & & & $\mathrm{x}$ & & & $\mathrm{x}$ & & 103 \\
\hline & & & & & & & & & & & & & & & $\mathrm{x}$ & & & $\mathrm{x}$ & $\mathrm{x}$ & 104 \\
\hline & & & & & & & & & & & & & & & $x$ & & & $x$ & & 38 \\
\hline Euphorbia pulcherrima & & & & & & & & & & & & & & & $\mathrm{x}$ & & & $\mathrm{x}$ & $\mathrm{x}$ & 105 \\
\hline & & & & & & & & & & & & & & & $\mathrm{x}$ & & & $\mathrm{x}$ & & 50 \\
\hline & & & & & & & & & & & & & & & $x$ & & & & & 106 \\
\hline & & & & & & & & & & & & & & & & $\mathrm{x}$ & & $\mathrm{x}$ & $\mathrm{x}$ & 107 \\
\hline Eustoma & & & & & & & & & & & & & & & $\mathrm{x}$ & & & $\mathrm{x}$ & & 108 \\
\hline & & & & & & & & & & & & & & & $x$ & & & $x$ & & 109 \\
\hline & & & & & & & & & & & & & & & $\mathrm{x}$ & & & $\mathrm{x}$ & & 34 \\
\hline & & & & & & & & & & & & & & & $\mathrm{x}$ & & & $\mathrm{x}$ & & 110 \\
\hline Ficus lurata & & & & & & & & & & & & & & & $\mathrm{x}$ & & & & $\mathrm{x}$ & 111 \\
\hline Ficus iyraca & & & & & & & & & & & & & & & $\mathrm{x}$ & & & $\mathrm{x}$ & $\mathrm{x}$ & 112 \\
\hline Forsythia & & & & & & & & & & & & & & & $\mathrm{x}$ & & & $\mathrm{x}$ & & 113 \\
\hline & & & & & & & & & & & & & & & & $\mathrm{x}$ & & $\mathrm{x}$ & $\mathrm{x}$ & 107 \\
\hline & & & & & & & & & & & & & & & $\mathrm{x}$ & & & & & 114 \\
\hline Gentiana & & & & & & & & & & & & & & & $\mathrm{x}$ & & & & $\mathrm{x}$ & 115 \\
\hline & & & & & & & & & & & & & & & $x$ & & & & $x$ & 116 \\
\hline & & & & & & & & & & & & & & & $\mathrm{x}$ & & & & & 117 \\
\hline Gerbera & & & & & & & & & & & & & & & $\mathrm{x}$ & & & $\mathrm{x}$ & & 118 \\
\hline Geriver a & & & & & & & & & & & & & & & $\mathrm{x}$ & $\mathrm{x}$ & & $\mathrm{x}$ & & 119 \\
\hline & & & & & & & & & & & & & & & & $x$ & & $x$ & & 120 \\
\hline Gladiolus & & & & & & & & & & & & & & & & $x$ & & $\mathrm{x}$ & $\mathrm{x}$ & 121 \\
\hline Gidurotus & & & & & & & & & & & & & & & & $x$ & & $\mathrm{x}$ & $\mathrm{x}$ & 122 \\
\hline & & & & & & & & & & & & & & & 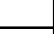 & $x$ & & $x$ & $\mathrm{x}$ & 20 \\
\hline Gypsophila & & & & & & & & & & & & & & & $x$ & & & $x$ & & 43 \\
\hline Hemerocallis & & & & & & & & & & & & & & & & $\mathrm{x}$ & & & & 123 \\
\hline Hibiscus & & & & & & & & & & & & & & & $\mathrm{x}$ & & & $x$ & & 124 \\
\hline & & & & & & & & & & & & & & & & & $\mathrm{x}$ & & & 66 \\
\hline Ipomoea & & & & & & & & & & & & & & & & & $\mathrm{x}$ & & & 67 \\
\hline & & & & & & & & & & & & & & & & & $\mathrm{x}$ & & & 68 \\
\hline & & & & & & & & & & & & & & & $\mathrm{x}$ & & & & & 125 \\
\hline & & & & & & & & & & & & & & & $x$ & & & $\mathrm{x}$ & & 126 \\
\hline Kalanchoe & & & & & & & & & & & & & & & $\mathrm{x}$ & & & $x$ & & 127 \\
\hline |Kalanenoe & & & & & & & & & & & & & & & $x$ & & & $x$ & $\mathrm{x}$ & 41 \\
\hline & & & & & & & & & & & & & & & $x$ & & & & & 56 \\
\hline & & & & & & & & & & & & & & & $\mathrm{x}$ & & & $\mathrm{x}$ & & 60 \\
\hline Lavatera & & & & & & & & & & & & & & & $\mathrm{x}$ & & & $\mathrm{x}$ & & 124 \\
\hline & & & & & & & & & & & & & & & $\mathrm{x}$ & & & $x$ & $\mathrm{x}$ & 128 \\
\hline & & & & & & & & & & & & & & & $\mathrm{x}$ & & & $\mathrm{x}$ & $\mathrm{x}$ & 8 \\
\hline & & & & & & & & & & & & & & & & $\mathrm{x}$ & & $\mathrm{x}$ & & 26 \\
\hline Lilium & & & & & & & & & & & & & & & $\mathrm{x}$ & & & $x$ & & 10 \\
\hline - & & & & & & & & & & & & & & & $\mathrm{x}$ & & & $x$ & & 129 \\
\hline & & & & & & & & & & & & & & & $x$ & & & $x$ & $x$ & 130 \\
\hline & & & & & & & & & & & & & & & & $\mathrm{x}$ & & $x$ & $x$ & 19 \\
\hline & & & & & & & & & & & & & & & $\mathrm{x}$ & & & $x$ & & 131 \\
\hline & & & & & & & & & & & & & & & & $\mathrm{x}$ & & $\mathrm{x}$ & & 132 \\
\hline & & & & & & & & & & & & & & & $\mathrm{x}$ & & & $x$ & & 36 \\
\hline & & & & & & & & & & & & & & & & $\mathrm{x}$ & & $\mathrm{x}$ & & 133 \\
\hline & & & & & & & & & & & & & & & & $\mathrm{x}$ & & $\mathrm{x}$ & $\mathrm{x}$ & 134 \\
\hline & & & & & & & & & & & & & & & $\mathrm{x}$ & & & $\mathrm{x}$ & $\mathrm{x}$ & 135 \\
\hline Orchidaceae & & & & & & & & & & & & & & & & $\mathrm{x}$ & & $\mathrm{x}$ & $\mathrm{x}$ & 136 \\
\hline & & & & & & & & & & & & & & & $\mathrm{x}$ & & & $x$ & & 54 \\
\hline & & & & & & & & & & & & & & & $x$ & & & $x$ & $\mathrm{x}$ & 33 \\
\hline & & & & & & & & & & & & & & & $x$ & & & $x$ & $\mathrm{x}$ & 137 \\
\hline & & & & & & & & & & & & & & & $x$ & & & $x$ & & 35 \\
\hline & & & & & & & & & & & & & & & $x$ & & & $x$ & & 55 \\
\hline
\end{tabular}




\begin{tabular}{|c|c|c|c|c|c|c|c|c|c|c|c|c|c|c|c|c|c|c|c|c|c|}
\hline \multirow[b]{3}{*}{ Plant } & \multicolumn{15}{|c|}{ GM traits } & \multirow{2}{*}{\multicolumn{3}{|c|}{ Transformation }} & \multirow{2}{*}{\multicolumn{2}{|c|}{$\begin{array}{l}\text { Transgene } \\
\text { sequences }\end{array}$}} & \multirow[b]{3}{*}{ References } \\
\hline & \multicolumn{9}{|c|}{ Biotic/abiotic stress resistance } & \multicolumn{6}{|c|}{ Plant attributes } & & & & & & \\
\hline & & 要 & 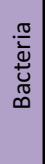 & $\stackrel{n}{\stackrel{n}{5}}$ & $\begin{array}{l}+ \\
\stackrel{5}{00} \\
0 \\
0 \\
0\end{array}$ & 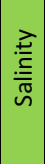 & $\frac{0}{0}$ & $\begin{array}{l}\stackrel{\tilde{J}}{\Xi} \\
\stackrel{I}{I}\end{array}$ & 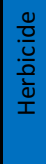 & $\frac{\grave{0}}{0}$ & 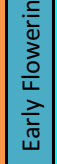 & 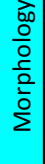 & 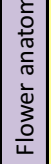 & 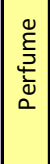 & 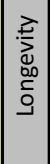 & 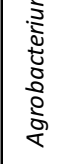 & 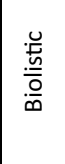 & 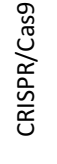 & 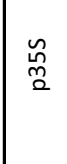 & $\sum_{i}^{n}$ & \\
\hline Ornithogalum & & & & & & & & & & & & & & & & $\mathrm{x}$ & & & $x$ & & 21 \\
\hline Osteospermum & & & & & & & & & & & & & & & & $\mathrm{x}$ & & & $\mathrm{x}$ & & 138 \\
\hline \multirow{4}{*}{ Pelargonium } & & & & & & & & & & & & & & & & $\mathrm{x}$ & & & $x$ & $\mathrm{x}$ & 139 \\
\hline & & & & & & & & & & & & & & & & $x$ & & & $x$ & $x$ & 59 \\
\hline & & & & & & & & & & & & & & & & $\mathrm{x}$ & & & $x$ & & 140 \\
\hline & & & & & & & & & & & & & & & & $x$ & & & & & 141 \\
\hline \multirow{25}{*}{ Petunia } & & & & & & & & & & & & & & & & $\mathrm{x}$ & & & $x$ & & 142 \\
\hline & & & & & & & & & & & & & & & & $\mathrm{x}$ & & & $\mathrm{x}$ & - & 143 \\
\hline & & & & & & & & & & & & & & & & $\mathrm{x}$ & & & & $\mathrm{x}$ & 9 \\
\hline & & & & & & & & & & & & & & & & $\mathrm{x}$ & & & $x$ & $\mathrm{x}$ & 144 \\
\hline & & & & & & & & & & & & & & & & $x$ & & & $x$ & & 145 \\
\hline & & & & & & & & & & & & & & & & $\mathrm{x}$ & & & $\mathrm{x}$ & & 146 \\
\hline & & & & & & & & & & & & & & & & $x$ & & & $\mathrm{x}$ & & 147 \\
\hline & & & & & & & & & & & & & & & & $x$ & & & & & 148 \\
\hline & & & & & & & & & & & & & & & & $\mathrm{x}$ & & & 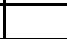 & & 149 \\
\hline & & & & & & & & & & & & & & & & $x$ & & & $x$ & & 126 \\
\hline & & & & & & & & & & & & & & & & $\mathrm{x}$ & & & & & 150 \\
\hline & & & & & & & & & & & & & & & & $\mathrm{x}$ & & & $x$ & $x$ & 80 \\
\hline & & & & & & & & & & & & & & & & $x$ & & & & & 151 \\
\hline & & & & & & & & & & & & & & & & $\mathrm{x}$ & & & $x$ & $x$ & 152 \\
\hline & & & & & & & & & & & & & & & & $\mathrm{x}$ & & & $\mathrm{x}$ & & 12 \\
\hline & & & & & & & & & & & & & & & & $x$ & & & $x$ & $x$ & 130 \\
\hline & & & & & & & & & & & & & & & & $\mathrm{x}$ & & & $x$ & & 153 \\
\hline & & & & & & & & & & & & & & & & $x$ & & & $\mathrm{x}$ & $\mathrm{x}$ & 154 \\
\hline & & & & & & & & & & & & & & & & $\mathrm{x}$ & & & $\mathrm{x}$ & $x$ & 155 \\
\hline & & & & & & & & & & & & & & & & $\mathrm{x}$ & & & $\mathrm{x}$ & & 156 \\
\hline & & & & & & & & & & & & & & & & $x$ & & & $x$ & $x$ & 157 \\
\hline & & & & & & & & & & & & & & & & $\mathrm{x}$ & & & $\mathrm{x}$ & $\mathrm{x}$ & 158 \\
\hline & & & & & & & & & & & & & & & & $x$ & & & & & 159 \\
\hline & & & & & & & & & & & & & & & & $x$ & & & $x$ & $x$ & 160 \\
\hline & & & & & & & & & & & & & & & & & & $x$ & & & 69 \\
\hline \multirow{11}{*}{ Rosa } & & & & & & & & & & & & & & & & $x$ & & & $x$ & & 52 \\
\hline & & & & & & & & & & & & & & & & $x$ & & & $x$ & & 25 \\
\hline & & & & & & & & & & & & & & & & $x$ & & & $\mathrm{x}$ & $\mathrm{x}$ & 161 \\
\hline & & & & & & & & & & & & & & & & $x$ & & & $x$ & & 162 \\
\hline & & & & & & & & & & & & & & & & $\mathrm{x}$ & & & $\mathrm{x}$ & $\mathrm{x}$ & 29 \\
\hline & & & & & & & & & & & & & & & & $\mathrm{x}$ & & & $\mathrm{X}$ & $x$ & 163 \\
\hline & & & & & & & & & & & & & & & & & $\bar{x}$ & & $x$ & $x$ & 164 \\
\hline & & & & & & & & & & & & & & & & $x$ & & & $x$ & & 165 \\
\hline & & & & & & & & & & & & & & & & $x$ & & & $x$ & & 44 \\
\hline & & & & & & & & & & & & & & & & $x$ & & & & & 166 \\
\hline & & & & & & & & & & & & & & & & $\mathrm{x}$ & & & $x$ & & 63 \\
\hline Sinningia speciosa & & & & & & & & & & & & & & & & $x$ & & & $x$ & & 167 \\
\hline & & & & & & & & & & & & & & & & $\mathrm{x}$ & & & $\mathrm{x}$ & $x$ & 168 \\
\hline & & & & & & & & & & & & & & & & $x$ & & & & $x$ & 169 \\
\hline & & & & & & & & & & & & & & & & $x$ & & & & $x$ & 170 \\
\hline & & & & & & & & & & & & & & & & $x$ & & & $x$ & $x$ & 171 \\
\hline Torenia & & & & & & & & & & & & & & & & $x$ & & & $x$ & $x$ & 48 \\
\hline & & & & & & & & & & & & & & & & & & $x$ & $x$ & & 70 \\
\hline & & & & & & & & & & & & & & & & $x$ & & & $x$ & & 172 \\
\hline & & & & & & & & & & & & & & & & $x$ & & & & $x$ & 49 \\
\hline & & & & & & & & & & & & & & & & $x$ & & & $\mathrm{x}$ & $x$ & 173 \\
\hline Tricyrtis & & & & & & & & & & & & & & & & $x$ & & & $\mathrm{X}$ & $x$ & 174 \\
\hline
\end{tabular}


Table 2 Representation (\%) of GM ornamental plants in scientific publications.

\begin{tabular}{|c|c|}
\hline Plants & $\%$ \\
\hline Anthurium & 0.6 \\
\hline Campanula & 1.2 \\
\hline Celosia & 0.6 \\
\hline Chrysanthemum & 26.7 \\
\hline Cyclamen & 0.6 \\
\hline Dianthus & 5.5 \\
\hline Euphorbia pulcherrima & 0.6 \\
\hline Eustoma & 4.2 \\
\hline Ficus lyrata & 1.2 \\
\hline Forsythia & 0.6 \\
\hline Gentiana & 3.0 \\
\hline Gerbera & 1.2 \\
\hline Gladiolus & 2.4 \\
\hline Gypsophila & 0.6 \\
\hline Hemerocallis & 0.6 \\
\hline Hibiscus & 0.6 \\
\hline Ipomoea & 1.8 \\
\hline Kalanchoe & 3.6 \\
\hline Lavatera & 0.6 \\
\hline Lilium & 4.8 \\
\hline Orchidaceae & 6.7 \\
\hline Ornithogalum & 0.6 \\
\hline Osteospermum & 0.6 \\
\hline Pelargonium & 2.4 \\
\hline Petunia & 15.2 \\
\hline Rosa & 6.7 \\
\hline Sinningia speciosa & 0.6 \\
\hline Torenia & 5.5 \\
\hline Tricyrtis & 0.6 \\
\hline
\end{tabular}

flowers make the product unmarketable or unacceptable for export ${ }^{4}$.

\section{Abiotic stress resistance}

Production of ornamental plants can be affected by abiotic stresses, such as drought, salinity, cold, heat, herbicides, etc. These stresses can affect plant growth and productivity as they generally damage the cellular machinery by inducing physiological, biochemical, and molecular changes in the plants ${ }^{5}$. Research on GM ornamentals has been conducted to improve abiotic
Table 3 Representation (\%) of GM traits in scientific publications.

\begin{tabular}{ll}
\hline GM traits & $\%$ \\
\hline Pest resistance & 5.5 \\
Fungi resistance & 7.9 \\
Bacteria resistance & 3.0 \\
Virus resistance & 7.9 \\
Drought resistance & 4.2 \\
Salinity resistance & 3.0 \\
Cold resistance & 4.8 \\
Heat & 1.2 \\
Herbicides & 2.4 \\
Color & 29.1 \\
Early flowering & 8.5 \\
Morphology & 12.7 \\
Flower anatomy & 4.8 \\
Perfume & 5.5 \\
Longevity & 12.1 \\
Several traits & 10.3 \\
\hline
\end{tabular}

stress resistance. Genes targeted for genetic transformation code for stress-protecting compounds have been classified into three categories: (i) genes coding the synthesis of various osmolytes such as mannitol, glycine betain, proline, and heat shock proteins, (ii) genes responsible for ion and water uptake and transport like aquaporins and ion transporter, etc., and (iii) genes regulating transcriptional controls and signal transduction mechanism, such as MAPK, DREBI, etc. ${ }^{5}$. As an example, overexpression of AtDREB1A gene increased drought and salt stress tolerance ${ }^{22}$, as does the overexpression of $C l C B F 1^{23}$ in transgenic chrysanthemum. The overexpression of the Chrysanthemum crassum plasma membrane $\mathrm{Na}+/ \mathrm{H}+$ antiporter gene CcSOS1 improved the salinity tolerance of chrysanthemum plants ${ }^{7}$. A construct carrying both CcSOS1 (from Chrysanthemum crassum) and CdICE1 (from Chrysanthemum dichrum) was constitutively expressed in the chrysanthemum variety "Jinba" ${ }^{24}$. Improved sensitivity to low temperature, drought, and salinity was observed in transgenic plants as measured by visible damage and plant survival ${ }^{24}$. The MtDREB1C gene, isolated from Medicago truncatula, enhanced freezing tolerance in transgenic China rose without morphological or developmental abnormality ${ }^{25}$. Resistance to herbicide was obtained in transgenic Lilium longiflorum expressing the bialaphos-resistance gene PAT under the constitutive CaMV35S promoter ${ }^{26}$. 


\section{Flower color modification}

Flower color is one of the most important traits of ornamental plants influencing its commercial value. Flower color can also attract pollinators and protect floral organs ${ }^{27}$. Several ornamental plants have been engineered for flower color modifications by targeting flavonoids, anthocyanins, carotenoids, and betalains ${ }^{28}$. To create new flower color, biosynthetic pathways can be modified through the introduction of new genes, overexpression, or silencing of target genes ${ }^{5}$. The first ornamental plant genetically modified for flower color was an orange pelargonidin-producing petunia variety ${ }^{12}$. This was achieved by the expression of the A1 gene from Zea mays L. encoding the enzyme dihydroflavonol reductase in a petunia plant defective for this gene ${ }^{12}$. Other examples concerned roses, carnations, and chrysanthemum which lack violet/blue varieties, due to the absence of delphinidin-based anthocyanins ${ }^{29}$. This is attributed to their deficiency of flavonoid $3^{\prime}, 5^{\prime}$-hydroxylase $\left(\mathrm{F}^{\prime} 5^{\prime} \mathrm{H}\right)$, a key enzyme in the synthesis of delphinidin ${ }^{29}$. Accumulation of delphinidin was achieved in roses or chrysanthemum by introducing the $\mathrm{F}^{\prime} 5^{\prime} \mathrm{H}$ gene, turning the flower color purple or violet ${ }^{29-31}$. Blue chrysanthemum was produced by introducing the $\mathrm{A}^{\prime} \mathrm{5}^{\prime} \mathrm{GT}$ gene encoding anthocyanin $3^{\prime}, 5^{\prime}$-O-glucosyltransferase, in addition to $\mathrm{F}^{\prime} 5^{\prime} \mathrm{H}$, into the host plant ${ }^{31}$.

\section{Early flowering}

Flowering time is also an important trait of ornamental plants which can been engineered. Several reports have described successful gene introduction to produce flowers in comparatively short time, allowing the production of flowers at a lower cost ${ }^{32}$. MADS box genes constitute an example as they can control flowering time and floral organ development ${ }^{3}$. Overexpression of AP1-like genes, member of MADS box gene family, from Asteraceae induced early-flowering in transgenic chrysanthemum plants $^{32}$. Transgenic Dendrobium orchids overexpressing DOAP1, an AP1 ortholog, displayed earlier flowering and earlier termination of inflorescence meristems into floral meristems than wild-type orchids ${ }^{33}$. Transgenic lisianthus plants, transformed with the MADS box gene OMADS1 from orchid flowered significantly earlier than nontransgenic plants ${ }^{34}$. Similarly, overexpression of MADS box genes, like DOSOC1 or OMADS1, promoted early flowering in transgenic orchids ${ }^{35,36}$.

\section{Morphology modification}

Plant morphology can be engineered to provide economic advantages during commercial production of ornamental plants. Indeed, the manipulation of plant size and development can produce plants with uniform morphology, and reduce the use of chemical growth regulators thereby reducing production $\operatorname{costs}^{37}$. Genetic engineering of homeotic, Agrobacterium, phytochrome, and gibberellin genes has provided potential targets to modify and control plant growth and development ${ }^{38}$. A dwarf chrysanthemum was produced through heterologous expression of the mutant Arabidopsis gai (gibberellic acid insensitive) gene driven from its own promoter by decreasing gibberellin response ${ }^{39}$. Ectopic expression of a tobacco (Nicotiana tabacum L.) phytochrome B1 gene in chrysanthenum modified plant architecture; GM plants were shorter with larger branch angles than wild-type plants ${ }^{37}$. A miniature chrysanthemum was produced through RNAi expression vector targeting both DmCPD and DmGA20ox, which are related to brassinosteroids (BR) and gibberellins (GA) biosynthesis, respectively ${ }^{40}$. Production of compact plants of Kalanchoe was achieved by overexpression of homeotic genes KxhKN4 and KxhKN5 ${ }^{41}$. The development of non-branching plants can reduce manual labor requirements during cultivation, decreasing the cost of flower production ${ }^{42}$. Transgenic gypsophila, carnation, or rosa exhibiting improved rooting were generated by expressing Agrobacterium rhizogenes ROL genes ${ }^{38,43,44}$. Enhancement of branching phenotype was observed in chrysanthemum by expression of the cytokinin biosynthesis gene ipt (isopentenyl transferase) ${ }^{45}$.

\section{Flower anatomy modification}

Ornamental plants have been engineered to create new flower shapes that can increase their ornamental value. Flower formation involves the development of sepals, petals, stamens, and pistils, regulated by several genes involved in flower organ identity ${ }^{34}$. The overexpression of CmTCP20, a member of teosinte branched1/cycloidea/proliferating cell factors (TCPs) gene family, led to larger flower inflorescences and longer petals in chrysanthemum ${ }^{46}$. Overexpression of CmCYC2c in Chrysanthemum lavandulifolium led to significant increase in flower numbers and petal ligule length of ray florets ${ }^{47}$. Alteration of flower transition and formation was observed in transgenic Eustoma grandiflorum plants ectopically expressing the MADS box gene LMADS1-M from lily (Lilium longiflorum $)^{34}$. Other studies have reported the modification of flower traits in transgenic torenia using chimeric repressors of Arabidopsis AGSRDX $^{48}$ or $\mathrm{TCP}^{49}$.

\section{Perfume modification}

Floral scent volatiles are implicated in the evolutionary success of many plants as they attract pollinators and seed dispersers. Most fragrance compounds belong to three major groups: terpenoid, phenylpropanoid/benzenoid, and aromatic amino acid $^{3}$. Most modern cut-flowers such as roses and carnations lack fragrance, probably because of the selection for other traits by breeders. Genetic 
engineering of ornamental plants could allow to transfer fragrance from one species to another, to induce new fragrance or enhance poor fragrance ${ }^{4}$. Fragrance in petals of transgenic lisianthus (Eustoma grandiflorum) was induced by overexpressing the Clarkia breweri BEAT gene (benzyl alcohol acetyl transferase) and fedding the transformed plants with alcoholic substrates ${ }^{50}$. Carnation plants were transformed with the linalool synthase (lis) gene from Clarkia breweri leading to the production of linalool and its derivatives in the transgenic plants ${ }^{51}$. In another study, an increase in the production of volatile phenylpropanoid/benzenoid compounds was observed in transgenic petunia flowers transformed with the Production of Anthocyanin Pigment1 (Pap1) Myb transcription factor from Arabidopsis thaliana ${ }^{43}$. Similarly, Pap1transgenic rosa flowers showed increased levels of volatile phenylpropanoid and terpenoid compounds when compared with control flowers ${ }^{52}$.

\section{Longevity}

Yellowing of leaves in ornamental plants, associated with senescence, have a negative attribute decreasing plant attractiveness, quality, and vase life ${ }^{3}$. In cut flowers, long vase life is a critical trait as they must survive several weeks before reaching the market ${ }^{4}$. Ethylene is a plant hormone, which plays a major role in the senescence process of plants ${ }^{53}$. Resistance to ethylene or inhibition of ethylene biosynthesis genes can increase shelf life ${ }^{3}$. Several ornamental plants have been transformed using genes encoding key enzymes of the ethylene pathway in order to inhibit senescence ${ }^{53}$. Expression of a mutated ethylene receptor gene etr1-1 from $A$. thaliana reduced ethylene sensibility in several ornamental plants, including orchid species $^{54,55}$, Campanula carpatica ${ }^{56,57}$, chrysanthemum ${ }^{58}$, carnation $^{53}$, Pelargonium zonale ${ }^{59}$, and Kalanchoe blossfeldian $a^{56,60}$. Similarly, expression of a mutated ethylene receptor gene (mDG-ERS1s) reduced ethylene sensitivity in modified chrysanthemum ${ }^{61}$. Transgenic carnation plants containing an antisense ACC oxidase gene exhibited low ethylene production and delayed petal senescence $^{62}$. An alternative approach to delay senescence in plants is to promote an increase in cytokinin levels ${ }^{59}$. Overproduction of cytokinins in petunia flowers transformed with PSAG12-IPT, enzyme which catalyzes the first step of the cytokinins biosynthesis pathway, delayed flower senescence, and decreased sensitivity to ethylene ${ }^{9}$. Delayed leaf senescence and enhanced resistance to exogenous ethylene were observed in miniature rose transformed with the same gene ${ }^{63}$.

\section{Detection of GM ornamentals}

When available, information on the transgene has been taken into account to provide an indication of the detectability of the events using common molecular biology tools (Table 1). Among the constructs, at least $83.0 \%$ present one of the screening sequences $\mathrm{p} 35 \mathrm{~S}$ or tNos commonly targeted in GMO detection strategies and $35.8 \%$ present both. If tested, these plants would therefore be identified as genetically modified.

\section{Conclusion}

The aim of this review was to list scientific publications related to GM ornamental plants developed by research groups and potentially presenting a commercial interest and to investigate if such unauthorized material would be detectable by laboratories testing for the presence of GM plants if unintentionally released on the marketplace. The goal was not to be exhaustive but to be as representative as possible of the constructs developed for research purpose. With 166 publications considered, it provides an overview of the constructs which could unintentionally reach the market without preliminary approval. The first conclusion of this review concerned the number of transformed ornamental species and the number of publications found per species. If many species have been transformed, two species of strong interest, chrysanthemum, and petunia, represent $>40 \%$ of the collected data. With no consideration of any health, or environmental risk, of trade or commercial importance, these two species represent higher risks of unintentional use simply by the number of constructs available. The second outcome of the review is the ability of laboratories to detect at least $83.0 \%$ of the identified constructs.

This review also outlined the diversity of GM traits of potential commercial interest for the producers and/or consumers studied. Color modification dominates the GM ornamental research, however, since the first transgenic color-modified carnation variety released $\sim 20$ years ago, very few GM ornamental varieties have been field tested and have obtained the regulatory approval. Indeed, with the exception of color-modified varieties of carnation and rose, no transgenic varieties of flowers have reached the market so far. The cost and time required to obtain the regulatory approval are the major factors limiting the commercialization of such plants and the profitability of such developments.

Whole-genome sequencing of ornamental plants and the development of new molecular markers allow the identification of new genes of interest and related pathways ${ }^{3}$. Considering the ease of use of the new genome editing tools $^{64}$ and especially of the CRISPR/Cas9 system $^{65}$, more and more transformed material will be available. To our knowledge, there are five scientific publications related to successful genome editing of ornamental plants with trait of commercial interest, including ipomoea ${ }^{66-68}$, petunia ${ }^{69}$, and torenia ${ }^{70}$. CRISPR/Cas9-mediated mutagenesis of the EPHEMERAL1 locus delayed petal senescence in transformed ipomoea ${ }^{66}$. Flower color changes was induced using 
CRISPR/Cas9 technology ${ }^{67,68,70}$. As an example, ipomoea with pale-yellow petals was produced for the first time by successful knockout of InCCD4, involved in carotenoid degradation, using CRISP/Cas9 system $^{68}$. Recently, the Court of Justice of the European Union (CJEU judgment of 25 July 2018, C-528/16) clarified that organisms obtained by new breeding techniques (NBT) are GMOs and fall within the scope of the EU GMO legislation. Meanwhile, several countries exporting crops to the EU (e.g., USA and Argentina) decided to not regulate such organisms as GMOs. Soon, it is probable that numerous varieties obtained by NBT will be available on the market outside of the EU, without any notification procedures in most countries. Accidental imports might occur between countries having different regulatory practices and policies regarding NBTs and environmental risk contamination should be considered.

Furthermore, groups will probably reduce the use of constructs including easily detectable promoters and terminators. The detectability of the produced transformed events will probably decrease in the near future; however, these considerations are not specific to ornamental plants.

\section{Author contributions}

A.L.B., N.D., D.P. and M.R. prepared Table 1. A.L.B. and M.R. wrote the paper

\section{Conflict of interest}

The authors declare that they have no conflict of interests.

Received: 19 July 2019 Revised: 20 November 2019 Accepted: 11 December 2019

Published online: 01 February 2020

\section{References}

1. Azadi, P., Bagheri, H., Nalousi, A. M., Nazari, F. \& Chandler, S. F. Current status and biotechnological advances in genetic engineering of ornamental plants. Biotechnol. Adv. 34, 1073-1090 (2016).

2. Kishi-Kaboshi, M., Aida, R. \& Sasaki, K. Genome engineering in ornamental plants: current status and future prospects. Plant Physiol. Biochem. 131, 47-52 (2018).

3. Noman, A. et al. Biotechnological advancements for improving floral attributes in ornamental plants. Front. Plant Sci. 8, 530 (2017).

4. Chandler, S. F. \& Sanchez, C. Genetic modification; the development of transgenic ornamental plant varieties. Plant Biotechnol. J. 10, 891-903 (2012).

5. Parmar, N. et al. Genetic engineering strategies for biotic and abiotic stress tolerance and quality enhancement in horticultural crops: a comprehensive review. 3 Biotech. 7, 239 (2017)

6. Darqui, F. S., Radonic, L. M., Hopp, H. E. \& Bilbao, M. L. Biotechnological improvement of ornamental plants. Ornam. Hortic. 23, 279-288 (2017).

7. An, J. et al. The over-expression of Chrysanthemum crassum CCSOS1 improves the salinity tolerance of chrysanthemum. Mol. Biol. Rep. 41, 4155-4162 (2014).

8. Azadi, P. et al. Increased resistance to cucumber mosaic virus (CMV) in Lilium transformed with a defective CMV replicase gene. Biotechnol. Lett. 33, 1249-1255 (2011).

9. Chang, H., Jones, M. L., Banowetz, G. M. \& Clark, D. G. Overproduction of cytokinins in petunia flowers transformed with P $_{\text {SAG12-IPT delays corolla }}$ senescence and decreases sensitivity to ethylene. Plant Physiol. 132, 2174-2183 (2003)

10. de Cáceres González, F. F. N., Davey, M. R., Sanchez, E. C. \& Wilson, Z. A. Conferred resistance to Botrytis cinerea in Lilium by overexpression of the RCH10 chitinase gene. Plant Cell Rep. 34, 1201-1209 (2015).
11. Hong, B. et al. Over-expression of AtDREB1A in chrysanthemum enhances tolerance to heat stress. Plant Mol. Biol. 70, 231-240 (2009).

12. Meyer, P., Heidmann, I., Forkmann, G. \& Saedler, H. A new petunia flower colour generated by transformation of a mutant with a maize gene. Nature 330, 677-678 (1987).

13. Meyer, P., Heidmann, I. \& Niedenhof, I. Differences in DNA-methylation are associated with a paramutation phenomenon in transgenic petunia. Plant $\mathrm{J}$. 4, 89-100 (1993).

14. Meyer, P. et al. Endogenous and environmental factors influence $35 \mathrm{~S}$ promoter methylation of a maize A1 gene construct in transgenic petunia and its colour phenotype. Mol. Gen. Genet. 231, 345-352 (1992).

15. Holst-Jensen, A. Testing for genetically modified organisms (GMOs): past, present and future perspectives. Biotechnol. Adv. 27, 1071-1082 (2009).

16. Bonfini, L., den Bulcke, Van, Mazzara, M. H., Ben, M. \& Patak, E. A. GMOMETHODS: the European Union database of reference methods for GMO analysis. J. AOAC Int. 95, 1713-1719 (2012).

17. Li, P. et al. The over-expression of a chrysanthemum WRKY transcription factor enhances aphid resistance. Plant Physiol. Biochem. 95, 26-34 (2015).

18. Valizadeh, M., Deraison, C., Kazemitabar, S. K., Rahbé, Y. \& Jongsma, M. A. Aphid resistance in florist's chrysanthemum (Chrysanthemum morifolium Ramat.) induced by sea anemone equistatin overexpression. Afr. J. Biotechnol. 12, 6922-6930 (2013).

19. Vieira, P. et al. Expression of a cystatin transgene can confer resistance to root lesion nematodes in Lilium lo.pnglorum cv. 'Nellie White'. Transgenic Res. 24 421-432 (2015).

20. Kamo, K. et al. Expression of a synthetic antimicrobial peptide, D4E1, in Gladiolus plants for resistance to Fusarium oxysporum f. sp. gladioli. Plant Cell Tiss. Organ Cult. 121, 459-467 (2015).

21. Lipsky, A., Joshi, J. R., Carmi, N. \& Yedidia, I. Expression levels of antimicrobial peptide tachyplesin I in transgenic Ornithogalum lines affect the resistance to Pectobacterium infection. J. Biotechnol. 238, 22-29 (2016).

22. Hong, B. et al. Heterologous expression of the AtDREB1A gene in chrysanthemum increases drought and salt stress tolerance. Sci. China C. Life Sci. 49, 436-445 (2006a).

23. Gao, W. et al. Overexpression of Chrysanthemum lavandulifolium CICBF1 in Chrysanthemum morifolium 'White Snow' improves the level of salinity and drought tolerance. Plant Physiol. Biochem. 124, 50-58 (2018).

24. Song, A. et al. The constitutive expression of a two transgene construct enhances the abiotic stress tolerance of chrysanthemum. Plant Physiol. Biochem. 80, 114-120 (2014).

25. Chen, J.-R. et al. DREBIC from Medicago truncatula enhances freezing tolerance in transgenic M. truncatula and China Rose (Rosa chinensis Jacq.). Plant Growth Regul. 60, 199-211 (2010).

26. Benedito, V. A. et al. Transformation of Lilium lo.pnglorum via particle bombardment and generation of herbicide-resistant plants. Crop Breed. Appl. Biotechnol. 5, 259-264 (2005).

27. Zhao, D. \& Tao, J. Recent advances on the development and regulation of flower color in ornamental plants. Front. Plant Sci. 6, 261 (2015).

28. Tanaka, Y. et al. Flower color modification by engineering of the flavonoid biosynthetic pathway: practical perspectives. Biosci. Biotechnol. Biochem. $\mathbf{7 4}$ 1760-1769 (2010)

29. Katsumoto, Y. et al. Engineering of the rose flavonoid biosynthetic pathway successfully generated blue-hued flowers accumulating delphinidin. Plant Cell Physiol. 48, 1589-1600 (2007).

30. Brugliera, F. et al. Violet/blue chrysanthemums-metabolic engineering of the anthocyanin biosynthetic pathway results in novel petal colors. Plant Cell Physiol. 54, 1696-1710 (2013).

31. Noda, N. et al. Generation of blue chrysanthemums by anthocyanin B-ring hydroxylation and glucosylation and its coloration mechanism. Sci. Adv. $\mathbf{3}$ e1602785 (2017)

32. Shulga, O. A., Mitiouchkina, T. Y., Shchennikova, A. V., Skryabin, K. G. \& Dolgov, S. V. Overexpression of AP1-like genes from Asteraceae induces earlyflowering in transgenic Chrysanthemum plants. Vitr. Cell. Dev. Biol. Plant 47 553-560 (2011).

33. Sawettalake, N., Bunnag, S., Wang, Y., Shen, L. \& Yu, H. DOAP1 promotes flowering in the orchid Dendrobium Chao Praya Smile. Front. Plant Sci. 8, 400 (2017).

34. Thiruvengadam, M. \& Yang, C.-H. Ectopic expression of two MADS box genes from orchid (Oncidium Gower Ramsey) and lily (Lilium longiflorum) alters 
flower transition and formation in Eustoma grandiflorum. Plant Cell Rep. 28, 1463-1473 (2009).

35. Thiruvengadam, M., Chung, I.-M. \& Yang, C.-H. Overexpression of Oncidium MADS box (OMADS1) gene promotes early flowering in transgenic orchid (Oncidium Gower Ramsey). Acta Physiol. Plant. 34, 1295-1302 (2012).

36. Ding, L., Wang, Y. \& Yu, H. Overexpression of DOSOC1, an ortholog of Arabidopsis SOC1, promotes flowering in the orchid Dendrobium Chao Parya Smile. Plant Cell Physiol. 54, 595-608 (2013).

37. Zheng, Z.-L., Yang, Z., Jang, J.-C. \& Metzger, J. D. Modification of plant architecture in chrysanthemum by ectopic expression of the tobacco phytochrome B1 gene. J. Am. Soc. Hortic. Sci. 126, 19-26 (2001).

38. Zuker, A. et al. RolC-transgenic carnation with improved horticultural traits: quantitative and qualitative analyses of greenhouse-grown plants. J. Am. Soc Hortic. Sci. 126, 13-18 (2001).

39. Petty, L. M., Harberd, N. P., Carré, I. A. Thomas, B. \& Jackson, S. D. Expression of the Arabidopsis gai gene under its own promoter causes a reduction in plant height in chrysanthemum by attenuation of the gibberellin response. Plant Sci. 164, 175-182 (2003).

40. Xie, Q., Chen, G., Liu, Q., Zhu, Z. \& Hu, Z. Dual silencing of DmCPD and DmGA20ox genes generates a novel miniature and delayed-flowering Dendranthema morifolium variety. Mol. Breed. 35, 67 (2015).

41. Lutken, $\mathrm{H}$. et al. Expression of KxhKN4 and KxhKN5 genes in Kalanchoe blossfeldiana 'Molly' results in novel compact plant phenotypes: towards a cisgenesis alternative to growth retardants. Plant Cell Rep. 30, 2267-2279 (2011).

42. Han, B. H., Suh, E. J., Lee, S. Y., Shin, H. K. \& Lim, Y. P. Selection of nonbranching lines induced by introducing Ls-like CDNA into Chrysanthemum (Dendranthema $\times$ grandiflorum (Ramat.) Kitamura) "Shuho-no-chikara". Sci. Hortic. 115, 70-75 (2007).

43. Moyal Ben Zvi, M. et al. Agrobacterium-mediated transformation of gypsophila (Gypsophila paniculata L.). Mol. Breed. 22, 543-553 (2008a).

44. van der Salm, T. P. M., van der Toorn, C. J. G., Bouwer, R., Hänisch ten Cate, C. H. \& Dons, H. J. M. Production of ROL gene transformed plants of Rosa hybrida L. and characterization of their rooting ability. Mol. Breed. 3, 39-47 (1997).

45. Khodakovskaya, M. et al. Enhancement of flowering and branching phenotype in chrysanthemum by expression of ipt under the control of a 0.821 kb fragment of the LEACO1 gene promoter. Plant Cell Rep. 28, 1351-1362 (2009).

46. Wang, J. et al. The CMTCP2O gene regulates petal elongation growth in Chrysanthemum morifolium. Plant Sci. 280, 248-257 (2019).

47. Huang, D. et al. Identification and characterization of CYC-like genes in regulation of ray floret development in Chrysanthemum morifolium. Front. Plant Sci. 7, 1633 (2016)

48. Narumi, T. et al. Chimeric AGAMOUS repressor induces serrated petal phenotype in Torenia fournieri similar to that induced by cytokinin application. Plant Biotechnol. 25, 45-53 (2008).

49. Sasaki, K., Yamaguchi, H., Kasajima, I., Narumi, T. \& Ohtsubo, N. Generation of novel floral traits using a combination of floral organ-specific promoters and a chimeric repressor in Torenia fournieri Lind. Plant Cell Physiol. 57, 1319-1331 (2016).

50. Aranovich, D., Lewinsohn, E. \& Zaccai, M. Post-harvest enhancement of aroma in transgenic lisianthus (Eustoma grandiflorum) using the Clarkia breweri benzyl alcohol acetyltransferase (BEAT) gene. Postharvest Biol. Tech. 43, 255-260 (2007).

51. Lavy, M. et al. Linalool and linalool oxide production in transgenic carnation flowers expressing the Clarkia breweri linalool synthase gene. Mol. Breed. 9, 103-111 (2002).

52. Moyal Ben Zvi, M. M. B. et al. PAP1 transcription factor enhances production of phenylpropanoid and terpenoid scent compounds in rose flowers. $N$. Phytol. 195, 335-345 (2012).

53. Bovy, A. G., Angenent, G. C., Dons, H. J. \& van Altvorst, A.-C. Heterologous expression of the Arabidopsis etri-1 allele inhibits the senescence of carnation flowers. Mol. Breed. 5, 301-308 (1999).

54. Raffeiner, B., Serek, M. \& Winkelmann, T. Agrobacterium tumefaciens-mediated transformation of Oncidium and Odontoglossum orchid species with the ethylene receptor mutant gene etr1-1. Plant Cell Tiss. Organ Cult. 98, 125-134 (2009).

55. Winkelmann, T., Warwas, M., Raffeiner, B., Serek, M. \& Mibus, H. Improved postharvest quality of inflorescences of fop 1::etr1-1 transgenic Burrageara 'Stefan Isler Lava Flow'. J. Plant Growth Regul. 35, 390-400 (2016).
56. Mibus, H., Sriskandarajah, S. \& Serek, M. Genetically modified flowering potted plants with reduced ethylene sensitivity. Acta Hortic. 847, 75-80 (2009).

57. Sriskandarajah, S., Mibus, H. \& Serek, M. Transgenic Campanula carpatica plants with reduced ethylene sensitivity. Plant Cell Rep. 26, 805-813 (2007).

58. Sumitomo, K, Narumi, T., Satoh, S. \& Hisamatsu, T. Involvement of the ethylene response pathway in dormancy induction in chrysanthemum. J. Exp. Bot. 59, 4075-4082 (2008).

59. Gehl, C., Wamhoff, D., Schaarschmidt, F. \& Serek, M. Improved leaf and flower longevity by expressing the etr1-1 allele in Pelargonium zonale under control of FBP1 and SAG12 promoters. Plant Growth Regul. 86, 351-363 (2018).

60. Sanikhani, M., Mibus, H., Stummann, B. M. \& Serek, M. Kalanchoe blossfeldiana plants expressing the Arabidopsis etr1-1 allele show reduced ethylene sensitivity. Plant Cell Rep. 27, 729-737 (2008).

61. Narumi, T., Aida, R., Ohmiya, A. \& Satoh, S. Transformation of chrysanthemum with mutated ethylene receptor genes: mDG-ERS1 transgenes conferring reduced ethylene sensitivity and characterization of the transformants. Postharvest. Biol. Tec. 37, 101-110 (2005).

62. Savin, K. W. et al. Antisense ACC oxidase RNA delays carnation petal senescence. HortScience 30, 970-972 (1995).

63. Zakizadeh, H., Lütken, H., Sriskandarajah, S., Serek, M. \& Müller, R. Transformation of miniature potted rose (Rosa hybrida cv. Linda) with $P_{\text {SAG } 2 \text {-ipt gene }}$ delays leaf senescence and enhances resistance to exogenous ethylene. Plant Cell Rep. 32, 195-205 (2013).

64. Mohanta, T. K., Bashir, T., Hashem, A., Abd_Allah, E. F. \& Bae, H. Genome editing tools in plants. Genes 8, 399 (2017).

65. Doudna, J. A. \& Charpentier, E. The new frontier of genome engineering with CRISPR-Cas9. Science 346, 1258096 (2014).

66. Shibuya, K, Watanabe, K. \& Ono, M. CRISPR/Cas9-mediated mutagenesis of the EPHEMERAL1 locus that regulates petal senescence in Japanese morning glory. Plant Physiol. Biochem. 131, 53-57 (2018).

67. Watanabe, $K$. et al. CRISPR/Cas9-mediated mutagenesis of the dihydroflavonol-4-reductase-B (DFR-B) locus in the Japanese morning glory Ipomoea (Pharbitis) nil. Sci. Rep. 7, 10028 (2017).

68. Watanabe, K., Oda-Yamamizo, C., Sage-Ono, K., Ohmiya, A. \& Ono, M. Alteration of flower colour in Ipomoea nil through CRISPR/Cas9-mediated mutagenesis of carotenoid cleavage dioxygenase 4. Transgenic Res. 27, 25-38 (2018).

69. Zhang, B., Yang, X., Yang, C., Li, M. \& Guo, Y. Exploiting the CRISPR/ Cas9 system for targeted genome mutagenesis in Petunia. Sci. Rep. 6, 20315 (2016).

70. Nishihara, M., Higuchi, A., Watanabe, A. \& Tasaki, K. Application of the CRISPR/ Cas9 system for modification of flower color in Torenia fournieri. BMC Plant Biol. 18, 331 (2018).

71. Kuehnle, A. R. et al. Peptide biocides for engineering bacterial blight tolerance and susceptibility in cut-flower Anthurium. Hort. Sci. 39, 1327-1331 (2004).

72. Sun, S.-B., Song, J.P. \& Yang, J. Overexpressing Arabidopsis KNAT1 gene in Celosia plumosus L. causes modification of plant morphology. Acta Physiol. Plant. 33, 1597-1602 (2011).

73. Aida, R., Komano, M., Saito, M., Nakase, K. \& Murai, K. Chrysanthemum flower shape modification by suppression of chrysanthemum-AGAMOUS gene. Plant Biotechnol. 25, 55-59 (2008).

74. Aswath, C. R., Mo, S. Y., Kim, S.H. \& Kim, D. H. IbMADS4 regulates the vegetative shoot development in transgenic chrysanthemum (Dendrathema grandiflora (Ramat.) Kitamura). Plant Sci. 166, 847-854 (2004).

75. Courtney-Gutterson, N. et al. Modification of flower color in florist's chrysanthemum: production of a white-flowering variety through molecular genetics. Bio/Technol. 12, 268-271 (1994).

76. Fan, Q. et al. CmWRKY1 enhances the dehydration tolerance of chrysanthemum through the regulation of ABA-associated genes. PLOS ONE 11, e0150572 (2016).

77. He, H., Ke, H., Keting, H., Qiaoyan, X. \& Silan, D. Flower colour modification of chrysanthemum by suppression of $F 3^{\prime} H$ and overexpression of the exogenous Senecio cruentus F3'5'H gene. PLOS ONE 8, e74395 (2013).

78. Hong, B. et al. Expression of the Arabidopsis DREBTA gene in transgenic chrysanthemum enhances tolerance to low temperature. J. Hortic. Sci. Biotech. 81, 1002-1008 (2006b).

79. Jiang, B. et al. The lateral suppressor-like gene, DgLSL, alternated the axillary branching in transgenic chrysanthemum (Chrysanthemum $x$ morifolium) by modulating IAA and GA content. Plant Mol. Biol. Rep. $\mathbf{2 8}$ 144-151 (2010) 
80. Khodakovskaya, M. et al. Effects of cor15a-IPT gene expression on leaf senescence in transgenic Petunia $\times$ hybrida and Dendranthema $\times$ grandiflorum. J. Exp. Bot. 56, 1165-1175 (2005).

81. Kim, Y.-S., Lim, S., Yoda, H., Choi, Y.-E. \& Sano, H. Simultaneous activation of salicylate production and fungal resistance in transgenic chrysanthemum producing caffeine. Plant Signal. Behav. 6, 409-412 (2011a).

82. Kim, Y.-S. et al. Resistance against beet armyworms and cotton aphids in caffeine-producing transgenic chrysanthemum. Plant Biotechnol. 28, 393-395 (2011b).

83. Kumar, S., Raj, S., Sharma, A. \& Varma, H. Genetic transformation and development of Cucumber mosaic virus resistant transgenic plants of Chrysanthemum morifolium cv. Kundan. Sci. Hortic. 134, 40-45 (2012).

84. Mao, Y. et al. Functional analysis of alternative splicing of the FLOWERING LOCUS T orthologous gene in Chrysanthemum morifolium. Hortic. Res. 3 16058 (2016)

85. Miao, Y., ZhaoLei, L., SuMei, C. \& FaDi, C. Expression of P. mume PGIP gene in transgenic Dendranthema morifolium increased tolerance to disease resistance. Acta Botanica Boreali.-Occidentalia Sin. 30, 1111-1116 (2010).

86. Satoh, S., Watanabe, M., Chisaka, K. \& Narumi, T. Suppressed leaf senescence in chrysanthemum transformed with a mutated ethylene receptor genemDG-ERST(etr1-4). J. Plant Biol. 51, 424 (2008).

87. Noda, N. et al. Genetic engineering of novel bluer-colored chrysanthemums produced by accumulation of delphinidin-based anthocyanins. Plant Cell Physiol. 54, 1684-1695 (2013).

88. Oda, A. et al. CSFTL3, a chrysanthemum FLOWERING LOCUS T-like gene, is a key regulator of photoperiodic flowering in chrysanthemums. J. Exp. Bot. 63 1461-1477 (2012).

89. Ogawa, T. et al. Double-stranded RNA-specific ribonuclease confers tolerance against chrysanthemum stunt viroid and tomato spotted wilt virus in transgenic chrysanthemum plants. Breed. Sci. 55, 49-55 (2005).

90. Ohmiya, A., Kishimoto, S., Aida, R., Yoshioka, S. \& Sumitomo, K. Carotenoid cleavage dioxygenase (CmCCD4a) contributes to white color formation in chrysanthemum petals. Plant Physiol. 142, 1193-1201 (2006).

91. Ohmiya, A., Sumitomo, K. \& Aida, R. "Yellow Jimba": suppression of carotenoid cleavage dioxygenase ( $\mathrm{CmCCD} 4 a$ ) expression turns white chrysanthemum petals yellow. J. Jpn. Soc. Hort. Sci. 78, 450-455 (2009).

92. Sen, S., Kumar, S., Ghani, M. \& Thakur, M. Agrobacterium mediated genetic transformation of chrysanthemum (Dendranthema grandiflora Tzvelev) with rice chitinase gene for improved resistance against Septoria obesa. Plant Pathol. J. 12, 1-10 (2013).

93. Shinoyama, H., Mochizuki, A., Komano, M., Nomura, Y. \& Nagai, T. Insect resistance in transgenic chrysanthemum [Dendranthema xgrandiflorum (Ramat.) Kitamura] by the introduction of a modified $\delta$-endotoxin gene of Bacillus thuringiensis. Breed. Sci. 53, 359-367 (2003).

94. Shinoyama, H. \& Mochizuki, A. Insect resistant transgenic chrysanthemum [Dendranthema X Grandiflorum (Ramat.) Kitamura]. Acta Hortic. 714, 177-184 (2006).

95. Shinoyama, H., Mochizuki, A., Nomura, Y. \& Kamada, H. Environmental risk assessment of genetically modified chrysanthemums containing a modified cry 1 Ab gene from Bacillus thuringiensis. Plant Biotechnol. 25, 17-29 (2008)

96. Shinoyama, H., Mitsuhara, I., Ichikawa, H., Kato, K. \& Mochizuki, A. Transgenic chrysanthemums (Chrysanthemum morifolium Ramat.) carrying both insect and disease resistance. Acta Hortic. 1087, 485-497 (2015).

97. Takatsu, Y., Nishizawa, Y., Hibi, T. \& Akutsu, K. Transgenic chrysanthemum (Dendranthema grandiflorum (Ramat.) Kitamura) expressing a rice chitinase gene shows enhanced resistance to gray mold (Botrytis cinerea). Sci. Hortic. 82, 113-123 (1999).

98. Xu, G., Chen, S. \& Chen, F. Transgenic chrysanthemum plants expressing a harpin $x_{0 o}$ gene demonstrate induced resistance to alternaria leaf spot and accelerated development. Russ. J. Plant Physiol. 57, 548-553 (2010).

99. Boase, M. R. et al. Isolation and antisense suppression of flavonoid 3', 5'hydroxylasemodifies flower pigments and colour in cyclamen. BMC Plant Biol. 10, 107 (2010).

100. Casanova, E. et al. ro/C-transgenic carnation plants: adventitious organogenesis and levels of endogenous auxin and cytokinins. Plant Sci. 167, 551-560 (2004)

101. Kosugi, Y. et al. Expression of genes responsible for ethylene production and wilting are differently regulated in carnation (Dianthus caryophyllus L.) petals. Plant Sci. 158, 139-145 (2000).
102. Meng, L.-S., Song, J.-P., Sun, S.-B. \& Wang, C.-Y. The ectopic expression of PttKN1 gene causes pleiotropic alternation of morphology in transgenic carnation (Dianthus caryophyllus L.). Acta Physiol. Plant. 31, 1155-1164 (2009).

103. Shirasawa-Seo, N. et al. Ectopic expression of an oat thionin gene in carnation plants confers enhanced resistance to bacterial wilt disease. Plant Biotechnol. 19, 311-317 (2002).

104. Zuker, A. et al. Modification of flower color and fragrance by antisense suppression of the flavanone 3-hydroxylase gene. Mol. Breed. 9, 33-41 (2002).

105. Clarke, J. L. et al. Agrobacterium tumefaciens-mediated transformation of poinsettia, Euphorbia pulcherrima, with virus-derived hairpin RNA constructs confers resistance to Poinsettia mosaic virus. Plant Cell Rep. 27, 1027-1038 (2008).

106. Deroles, S. C. et al. An antisense chalcone synthase cDNA leads to novel colour patterns in lisianthus (Eustoma grandiflorum) flowers. Mol. Breed. 4, 59-66 (1998).

107. Fekih, R., Yamagishi, N. \& Yoshikawa, N. Apple latent spherical virus vectorinduced flowering for shortening the juvenile phase in Japanese gentian and lisianthus plants. Planta 244, 203-214 (2016).

108. Nielsen, K. et al. Antisense flavonol synthase alters copigmentation and flower color in lisianthus. Mol. Breed. 9, 217-229 (2002).

109. Schwinn, K. E. et al. MYB and bHLH transcription factor transgenes increase anthocyanin pigmentation in petunia and lisianthus plants, and the petunia phenotypes are strongly enhanced under field conditions. Front. Plant Sci. $\mathbf{5}$, 603 (2014).

110. Zaccai, M., Lewinsohn, E. \& Pichersky, E. Modifying lisianthus traits by genetic engineering. Acta Hortic. 552, 137-142 (2001).

111. Amaro, J. T., Herrera, J. S. \& Hernández, A. Response of transgenic lines of Ficus lyrata Warb. carrying PSAG12:Ipt chimeric gene under different growing environments. Bioagro 24, 57-60 (2012).

112. Zhao, J. et al. Purple-leaved Ficus lyrata plants produced by overexpressing a grapevine VvMybA1 gene. Plant Cell Rep. 32, 1783-1793 (2013).

113. Rosati, C., Cadic, A., Duron, M., Renou, J.-P. \& Simoneau, P. Molecular cloning and expression analysis of dihydroflavonol 4-reductase gene in flower organs of Forsythia $\times$ intermedia. Plant Mol. Biol. 35, 303-311 (1997).

114. Nakatsuka, T. et al. Flower color modification of gentian plants by RNAimediated gene silencing. Plant Biotechnol. 25, 61-68 (2008).

115. Nakatsuka, T. et al. Genetic engineering of novel flower colour by suppression of anthocyanin modification genes in gentian. J. Plant Physiol. 167 231-237 (2010).

116. Nakatsuka, T., Saito, M., Yamada, E. \& Nishihara, M. Production of picotee-type flowers in Japanese gentian by CRES-T. Plant Biotechnology 28, 173-180 (2011).

117. Tasaki, K., Atsumi, G., Nishihara, M. \& Sekine, K.-T. Development of a Broad bean wilt virus 2-based expression vector for gentian. Sci. Hortic. 201, 279-286 (2016).

118. Korbin, M. Assessment of gerbera plants genetically modified with TSW nucleocapsid gene. J. Fruit. Ornam. Plant Res. 14, 1-9 (2006).

119. Laitinen, R. A., Ainasoja, M., Broholm, S. K., Teeri, T. H. \& Elomaa, P. Identification of target genes for a MYB-type anthocyanin regulator in Gerbera hybrida. J. Exp. Bot. 59, 3691-3703 (2008).

120. Kamo, K. et al. Transgenic Gladiolus plants transformed with the bean yellow mosaic virus coat-protein gene in either sense or antisense orientation. Plant Cell Rep. 23, 654-663 (2005).

121. Kamo, K., Jordan, R., Guaragna, M. A., Hsu, H. T. \& Ueng, P. Resistance to Cucumber mosaic virus in Gladiolus plants transformed with either a defective replicase or coat protein subgroup II gene from Cucumber mosaic virus. Plant Cell Rep. 29, 695-704 (2010).

122. Kamo, K. et al. Gladiolus plants transformed with single-chain variable fragment antibodies to Cucumber mosaic virus. Plant Cell Tiss. Org. 110, 13-21 (2012).

123. Aziz, A., Sauve, R. \& Zhou, S. Genetic transformation of Stella De Oro daylily by particle bombardment. Can. J. Plant Sci. 83, 873-876 (2003).

124. Vazquez-Thello, A., Yang, J. L., Hidaka, M. \& Uozumi, T. Inherited chilling tolerance in somatic hybrids of transgenic Hibiscus rosa-sinensis $x$ transgenic Lavatera thuringiaca selected by double-antibiotic resistance. Plant Cell Rep. 15, 506-511 (1996).

125. Christensen, B., Sriskandarajah, S., Serek, M. \& Muller, R. Transformation of Kalanchoe blossfeldiana with rol-genes is useful in molecular breeding towards compact growth. Plant Cell Rep. 27, 1485-1495 (2008). 
126. Gargul, J. M., Mibus, H. \& Serek, M. Manipulation of MKS1 gene expression affects Kalanchoe blossfeldiana and Petunia hybrida phenotypes. Plant Biotechnol. J. 13, 51-61 (2015).

127. Lutken, $\mathrm{H}$. et al. Production of compact plants by overexpression of AtSHI in the ornamental. Kalanchoe. Plant Biotechnol. J. 8, 211-222 (2010).

128. Azadi, P. et al. Metabolic engineering of Lilium $\times$ formolongi using multiple genes of the carotenoid biosynthesis pathway. Plant Biotechnol. Rep. 4, 269-280 (2010)

129. Kamo, K., Thilmony, R. \& Bauchan, G. Transgenic Lilium lo.pnglorum plants containing the bar-uidA gene controlled by the rice $R P C 1$, Agrobacterium rolD, mas2, and CaMV 355 promoters. Plant Cell Tiss. Org. 136, 303-312 (2019).

130. Qi, Y., Lou, Q., Quan, Y., Liu, Y. \& Wang, Y. Flower-specific expression of the Phalaenopsis flavonoid $3^{\prime}, 5^{\prime}$-hydoxylase modifies flower color pigmentation in Petunia and Lilium. Plant Cell Tiss. Org. 115, 263-273 (2013).

131. Yoshida, K., Oyama-Okubo, N. \& Yamagishi, M. An R2R3-MYB transcription factor ODORANT1 regulates fragrance biosynthesis in lilies (Lilium spp.). Mol. Breed. 38, 144 (2018)

132. Chang, $C$. et al. Transgenic resistance to Cymbidium mosaic virus in Dendrobium expressing the viral capsid protein gene. Transgenic Res. 14, 41-46 (2005).

133. Knapp, J. E., Kausch, A. P. \& Chandlee, J. M. Transformation of three genera of orchid using the bar gene as a selectable marker. Plant Cell Rep. 19, 893-898 (2000).

134. Kuehnle, A. R. \& Sugii, N. Transformation of Dendrobium orchid using particle bombardment of protocorms. Plant Cell Rep. 11, 484-488 (1992).

135. Liau, C.-H. et al. The sweet pepper ferredoxin-like protein (pflp) conferred resistance against soft rot disease in Oncidium orchid. Transgenic Res. 12, 329-336 (2003).

136. Liao, L.-J. et al. Transgene silencing in Phalaenopsis expressing the coat protein of Cymbidium Mosaic Virus is a manifestation of RNA-mediated resistance. Mol. Breed. 13, 229-242 (2004).

137. Sjahril, R. et al. Transgenic Phalaenopsis plants with resistance to Erwinia carotovora produced by introducing wasabi defensin gene using Agrobacterium method. Plant Biotechnol. 23, 191-194 (2006).

138. Giovannini, A., Zottini, M., Morreale, G., Spena, A. \& Allavena, A. Ornamental traits modification by rol genes in Osteospermum ecklonis transformed with Agrobacterium tumefaciens. Vitr. Cell. Dev. Biol. Plant 35, 70-75 (1999).

139. Bi, Y.-M., Cammue, B., Goodwin, P., KrishnaRaj, S. \& Saxena, P. Resistance to Botrytis cinerea in scented geranium transformed with a gene encoding the antimicrobial protein Ace-AMP1. Plant Cell Rep. 18, 835-840 (1999).

140. Kanemaki, A. et al. Ectopic expression of the R2R3-MYB gene from Tricyrtis sp. results in leaf color alteration in transgenic Pelargonium crispum. Sci. Hortic. 240, 411-416 (2018)

141. Saxena, G. et al. Rose-scented geranium (Pelargonium sp.) generated by Agrobacterium rhizogenes mediated Ri-insertion for improved essential oil quality. Plant Cell Tiss. Org. 90, 215-223 (2007).

142. Moyal Ben Zvi, M. et al. Interlinking showy traits: co-engineering of scent and colour biosynthesis in flowers. Plant Biotechnol. J. 6, 403-415 (2008b).

143. Boase, M. R. et al. Failure to launch: the self-regulating $M d-M Y B 1 O_{R 6}$ gene from apple is active in flowers but not leaves of Petunia. Plant Cell Rep. 34, 1817-1823 (2015).

144. Chin, D. P. et al. Generation of brilliant green fluorescent petunia plants by using a new and potent fluorescent protein transgene. Sci. Rep. 8, 16556 (2018).

145. Chu, Y. X., Chen, H. R., Wu, A. Z., Cai, R. \& Pan, J. S. Expression analysis of dihydroflavonol 4-reductase genes in Petunia hybrida. Genet. Mol. Res. 14, 5010-5021 (2015).

146. Davies, K. M., Bloor, S. J., Spiller, G. B. \& Deroles, S. C. Production of yellow colour in flowers: redirection of flavonoid biosynthesis in Petunia. Plant J. 13, 259-266 (1998).

147. Davies, K. M. et al. Enhancing anthocyanin production by altering competition for substrate between flavonol synthase and dihydroflavonol 4reductase. Euphytica 131, 259-268 (2003).

148. de Vetten, N. et al. A cytochrome b5 is required for full activity of flavonoid 3', 5'-hydroxylase, a cytochrome P450 involved in the formation of blue flower colors. Proc. Natl Acad. Sci. USA 96, 778-783 (1999).

149. Estrada-Melo, A. C., Ma, C., Reid, M. S. \& Jiang, C.-Z. Overexpression of an ABA biosynthesis gene using a stress-inducible promoter enhances drought resistance in petunia. Hortic. Res. 2, 15013 (2015).
150. Holton, T. A., Brugliera, F. \& Tanaka, Y. Cloning and expression of flavonol synthase from Petunia hybrida. Plant J. 4, 1003-1010 (1993).

151. Lee, K.-J., Kim, Y.-E., Lee, H. \& Park, S.-Y. Overexpression of SUMO E3 ligase HPY2 regulates the cell cycle in petunia development. Hortic. Environ. Biotechnol. 58, 384-392 (2017).

152. Lucker, J. et al. Expression of Clarkia S-linalool synthase in transgenic petunia plants results in the accumulation of S-linalyl-beta-D-glucopyranoside. Plant J. 27, 315-324 (2001)

153. Qi, Y., Yao, X., Zhao, D. \& Lu, L. Overexpression of SbSKIP, a pre-mRNA splicing factor from Sorghum bicolor, enhances root growth and drought tolerance in Petunia hybrida. Sci. Hortic. 240, 281-287 (2018).

154. Shah, D. M. et al. Engineering herbicide tolerance in transgenic plants. Science 233, 478-481 (1986)

155. Shaw, J.-F., Chen, H.-H., Tsai, M.-F., Kuo, C.-I. \& Huang, L.-C. Extended flower longevity of Petunia hybrida plants transformed with boers, a mutated ERS gene of Brassica oleracea. Mol. Breed. 9, 211-216 (2002).

156. Sun, D. et al. LrABCF1, a GCN-type ATP-binding cassette transporter from Lilium regale, is involved in defense responses against viral and fungal pathogens. Planta 244, 1185-1199 (2016).

157. Tsuda, S. et al. Flower color modification of Petunia hybrida commercial varieties by metabolic engineering. Plant Biotechnol. 21, 377-386 (2004).

158. Van der Krol, A. R. et al. An anti-sense chalcone synthase gene in transgenic plants inhibits flower pigmentation. Nature 333, 866-869 (1988).

159. Warner, R. M. Genetic approaches to improve cold tolerance of Petunia. Plant Breed. Genet. Eng. 306, 1-2 (2010).

160. Wilkinson, J. Q. et al. A dominant mutant receptor from Arabidopsis confers ethylene insensitivity in heterologous plants. Nat. Biotechnol. 15, 444-447 (1997).

161. Chen, J.-R. et al. Co-expression of MtDREB1C and RCXET enhances stress tolerance of transgenic China rose (Rosa chinensis Jacq.). J. Plant Growth Regul. 35, 586-599 (2016).

162. Dohm, A., Ludwig, C., Schilling, D. \& Debener, T. Transformation of roses with genes for antifungal proteins to reduce their susceptibility to fungal diseases. Acta Hortic. 572, 105-111 (2002).

163. Li, X., Gasic, K., Cammue, B., Broekaert, W. \& Korban, S. S. Transgenic rose lines harboring an antimicrobial protein gene, Ace-AMP1, demonstrate enhanced resistance to powdery mildew (Sphaerotheca pannosa). Planta 218, 226-232 (2003).

164. Marchant, R. et al. Expression of a chitinase transgene in rose (Rosa hybrida L.) reduces development of blackspot disease (Diplocarpon rosae Wolf). Mol. Breed. 4, 187-194 (1998).

165. Nakamura, N. et al. Flower color modification in Rosa hybrida by expressing the S-adenosylmethionine: anthocyanin 3',5'-O-methyltransferase gene from Torenia hybrida. Plant Biotechnology 32, 109-117 (2015).

166. Yan, H. et al. Graft-accelerated virus-induced gene silencing facilitates functional genomics in rose flowers. J. Integr. Plant Biol. 60, 34-44 (2018).

167. Li, X. et al. Flowering time control in ornamental gloxinia (Sinningia speciosa) by manipulation of miR159 expression. Ann. Bot. 111, 791-799 (2013).

168. Aida, R., Yoshida, T., Ichimura, K., Goto, R. \& Shibata, M. Extension of flower longevity in transgenic torenia plants incorporating ACC oxidase transgene. Plant Sci. 138, 91-101 (1998).

169. Aida, R., Kishimoto, S., Tanaka, Y. \& Shibata, M. Modification of flower color in torenia (Torenia fournieri Lind.) by genetic transformation. Plant Sci. 153, 33-42 (2000a).

170. Aida, R., Yoshida, K., Kondo, T., Kishimoto, S. \& Shibata, M. Copigmentation gives bluer flowers on transgenic torenia plants with the antisense dihydroflavonol-4-reductase gene. Plant Sci. 160, 49-56 (2000b).

171. Nakamura, N. et al. Generation of pink flower varieties from blue Torenia hybrida by redirecting the flavonoid biosynthetic pathway from delphinidin to pelargonidin. Plant Biotechnol. 27, 375-383 (2010).

172. Ueyama, Y. et al. Molecular and biochemical characterization of torenia flavonoid $3^{\prime}$-hydroxylase and flavone synthase $\|$ and modification of flower color by modulating the expression of these genes. Plant Sci. 163, 253-263 (2002).

173. Suzuki, K.-I. et al. Flower color modifications of Torenia hybrida by cosuppression of anthocyanin biosynthesis genes. Mol. Breed. 6, 239-246 (2000).

174. Kamiishi, Y. et al. Flower color alteration in the liliaceous ornamental Tricyrtis sp. by RNA interference-mediated suppression of the chalcone synthase gene. Mol. Breed. 30, 671-680 (2012). 